Meta

Journal des traducteurs

Translators' Journal

\title{
Jean-René Ladmiral - une anthropologie interdisciplinaire de la traduction
}

\section{Jane Elisabeth Wilhelm}

Volume 57, numéro 3, septembre 2012

URI : https://id.erudit.org/iderudit/1017079ar

DOI : https://doi.org/10.7202/1017079ar

Aller au sommaire du numéro

Éditeur(s)

Les Presses de l’Université de Montréal

ISSN

0026-0452 (imprimé)

1492-1421 (numérique)

Découvrir la revue

Citer ce document

Wilhelm, J. E. (2012). Jean-René Ladmiral - une anthropologie interdisciplinaire de la traduction. Meta, 57(3), 546-563.

https://doi.org/10.7202/1017079ar

\section{Résumé de l'article}

Jean-René Ladmiral est connu dans le monde de la traduction non seulement comme traductologue et traducteur de la philosophie allemande (en particulier de Jürgen Habermas et de l'École de Francfort, par ailleurs aussi de Kant et de Nietzsche), mais encore en tant que philosophe. À ce double titre, il a travaillé sur l'épistémologie des sciences humaines dans une perspective interdisciplinaire. En convoquant, dans cet entretien, l'apport des sciences psychologiques, et notamment de la psychanalyse, il examine les bases épistémologiques de la recherche en traductologie. À son sens, la philosophie, la psychanalyse et la traductologie sont trois disciplines réflexives, c'est-à-dire qu'elles font retour sur ce qu'on vit, sur ce qu'on pense et ce qu'on fait. Il plaide pour ce qu'il appelle une traductologie productive, qui s'intéresse non pas à la traduction déjà faite, mais au processus de traduction, d'où la référence à la psychologie. La traductologie se situerait ainsi à l'articulation de la linguistique et de la littérature comparée, surplombée par la philosophie avec un soubassement en psychologie. Ce sont toutes les sciences humaines et les études culturelles, en définitive, qui sont concernées par cette discipline, en sorte que la traductologie, pour Jean-René Ladmiral, tend à prendre l'ampleur d'une anthropologie interdisciplinaire de la traduction.
Ce document est protégé par la loi sur le droit d'auteur. L'utilisation des services d’Érudit (y compris la reproduction) est assujettie à sa politique d'utilisation que vous pouvez consulter en ligne.

https://apropos.erudit.org/fr/usagers/politique-dutilisation/ 


\title{
Jean-René Ladmiral - une anthropologie interdisciplinaire de la traduction
}

\author{
Entretien avec \\ JANE ELISABETH WILHELM \\ Université Sorbonne Nouvelle - Paris 3 \\ janewilhelm@bluewin.ch
}

\begin{abstract}
RÉSUMÉ
Jean-René Ladmiral est connu dans le monde de la traduction non seulement comme traductologue et traducteur de la philosophie allemande (en particulier de Jürgen Habermas et de l'École de Francfort, par ailleurs aussi de Kant et de Nietzsche), mais encore en tant que philosophe. À ce double titre, il a travaillé sur l'épistémologie des sciences humaines dans une perspective interdisciplinaire. En convoquant, dans cet entretien, l'apport des sciences psychologiques, et notamment de la psychanalyse, il examine les bases épistémologiques de la recherche en traductologie. À son sens, la philosophie, la psychanalyse et la traductologie sont trois disciplines réflexives, c'est-àdire qu'elles font retour sur ce qu'on vit, sur ce qu'on pense et ce qu'on fait. Il plaide pour ce qu'il appelle une traductologie productive, qui s'intéresse non pas à la traduction déjà faite, mais au processus de traduction, d'où la référence à la psychologie. La traductologie se situerait ainsi à l'articulation de la linguistique et de la littérature comparée, surplombée par la philosophie avec un soubassement en psychologie. Ce sont toutes les sciences humaines et les études culturelles, en définitive, qui sont concernées par cette discipline, en sorte que la traductologie, pour Jean-René Ladmiral, tend à prendre l'ampleur d'une anthropologie interdisciplinaire de la traduction.
\end{abstract}

\begin{abstract}
Jean-René Ladmiral is well-known in translation circles not only as a translation studies scholar and a translator of German philosophy (in particular of Jürgen Habermas and the Frankfurt School, but also of Kant and Nietzsche), and as a philosopher as well. As such, he has examined the epistemology of social sciences in an interdisciplinary perspective. In this interview, he calls on different schools of psychology and, in particular, on psychoanalysis to discuss the epistemological basis of research in translation studies. In his view, philosophy, psychoanalysis and translation studies are three reflexive disciplines, that is, they reflect on what one experiences, thinks or does. He speaks in favour of what he calls "une traductologie productive," whose interest lies not in analyzing existing translations but rather the process of translation, and this is why he makes reference to psychology. Translation studies would be situated at the junction of linguistics and comparative literature, with philosophy towering above and psychology as its base. All social sciences and cultural studies are ultimately related to this field of research. For Jean-René Ladmiral it tends to take on the scope of an interdisciplinary anthropology of translation.
\end{abstract}

\section{MOTS-CLÉS/KEY WORDS}

entretien, épistémologie de la traduction, psychologie, psychanalyse interview, epistemology of translation, psychology, psychoanalysis 
Jean-René Ladmiral est philosophe, linguiste et germaniste, traducteur et théoricien de la traduction. S'il est connu dans le monde de la traduction à la fois comme traductologue et traducteur de la philosophie allemande (en particulier de Jürgen Habermas et de l'École de Francfort, mais aussi de Kant et de Nietzsche), en tant que philosophe, il a travaillé sur l'épistémologie de la traduction dans une perspective interdisciplinaire. Plus généralement, l'horizon de ses recherches profile une anthropologie interdisciplinaire de la traduction. En convoquant l'apport des sciences psychologiques, et notamment de la psychanalyse, il examine ici les bases épistémologiques de la recherche en traductologie.

\section{- Comment se fait-il que vous vous soyez intéressé à la traduction?}

C'était effectivement inattendu dans la mesure où la traduction, aux yeux de beaucoup de monde, a longtemps été considérée comme une tâche "ancillaire». Il n'est que trop certain que, pour quelques personnes encore, la traduction est une activité tout à fait subalterne et, à la limite, moins valorisée qu'un travail de secrétariat. Pour sa part, Antoine Berman se gendarmait contre cette limitation de la traduction à une tâche ancillaire. Quand les gens n'y réfléchissent pas, ils se disent qu'on connaît des langues, donc on traduit. Un de mes collègues à l'Université de Paris X-Nanterre un jour m'a demandé: "J'ai trente pages d'un article, est-ce que tu veux bien me les traduire?» Alors, je lui ai répondu: «C'est du travail, ça prend du temps, ça coûte de l'argent!»Et j'ai vu dans son regard que je le décevais: en somme, Ladmiral n'était ni aussi sympathique, ni aussi serviable - ni même aussi compétent qu'il l'avait cru. Parce que trente pages, ça se fait comme ça! S'il m'avait demandé de taper trente pages, cela aurait peut-être eu encore plus de valeur à ses yeux. Il m'aurait donné un manuscrit de trente pages à dactylographier, il aurait compris que c'était un travail. En somme, la traduction, c'est un peu moins que la dactylographie: avec juste un petit problème de langue en plus, mais ça compte à peine. C'est pourquoi l'une des tâches importantes, pour ceux qui sont dans le domaine, c'est de faire la promotion de la traduction et de défendre le métier. Mais il reste qu'il est difficile de «vendre» le fait que c'est un travail.

En ce qui me concerne, j'ai pris conscience de la traduction très tôt pour différentes raisons. D’abord, nous en avons tous fait au lycée; et ensuite, je me suis beaucoup intéressé aux langues, surtout à l'allemand. J'ai eu un excellent professeur qui prenait le temps d'expliquer les choses, de créer un dialogue, ce qui a déclenché mon affection pour l'allemand, devenu ma première langue de travail. Ma combinaison linguistique, c'est français-allemand-anglais. La première traduction que j'ai publiée était Crisis of Psychoanalysis de Erich Fromm. Ce livre comportait une petite partie en allemand et une grande partie en anglais. Le rapport à une langue étrangère pose la question de la traduction implicitement: d'une façon ou d'une autre, on ne peut pas ne pas se poser la question du transfert linguistique. Lorsqu'on fait du transfert d'une langue à l'autre, ce n'est pas à proprement parler de la traduction, car on n'a pas encore compris les enjeux même de cette dernière, mais d'une certaine façon on est sur la voie. La deuxième raison qui m'a conduit à la traduction, c'est le rapport à la philosophie. 


\section{- C'est un peu paradoxal, pourriez-vous en dire un peu plus?}

Ce qui me fascinait dans l'allemand, c'était sa syntaxe très structurée; et j'anticipais sans doute déjà la philosophie. J'ai tendance à interpréter rétrospectivement, comme on le fait souvent, mon passé à la lumière de l'avenir. J'avais l'impression que cette syntaxe allemande complexe, mais en même temps structurée, me mettait sur la piste de la rationalité de la philosophie; et j'associais étroitement la philosophie et l'allemand. Je m’étais donné comme règle de ne jamais passer une année sans avoir été en Allemagne, ce qui m'a conduit à une bonne maîtrise de l'allemand. Les textes des philosophes allemands ne sont pas très faciles et, d'une certaine façon, on comprend un texte difficile quand on le traduit pour soi. C'est, donc, la deuxième raison qui m'a poussé à la traduction.

À cela vient s'ajouter qu'une des tâches du métier philosophique est de traduire les grands philosophes. Pour ma Maîtrise (DES), j’ai travaillé sur «Heidegger lecteur de Kant». Mais avant, j'avais traduit un texte de Wilhelm Reich, resté inédit, sur «le caractère masochiste». Cette première expérience de traduction de Wilhelm Reich s'articulait autour de la psychanalyse et de la politique, peu avant 68. En traduisant Reich, j'anticipais d'une certaine façon sur ce qui allait se produire. En somme, c'était un triangle: il y avait la philosophie, la politique, et puis la traduction. Et la traduction, c'était la mise à l'épreuve - dans le langage - des grandes idées, des grandes aspirations de la philosophie et de la politique. Dans mon esprit, sans réduire la traduction à une affaire sans enjeu, ni épaisseur, elle était pour moi tout de même subordonnée à la réflexion philosophique.

\section{- Quand a eu lieu votre rencontre avec la pensée et l'œuvre de Jürgen Habermas dont vous avez été le premier traducteur?}

On m’a très tôt proposé de traduire quelqu'un qui, à l'époque, était un inconnu, et qui était Jürgen Habermas. C'était un penseur important de l'École de Francfort, que ma fréquentation de l'Université allemande m'avait fait connaître. La façon pour moi de traiter de cet auteur, c'était de faire traduction et commentaire: la traduction étant le point de départ du commentaire, et le commentaire l'achèvement de la traduction. C'était un des aspects du métier philosophique. La traduction, dans cette affaire, était une des pratiques, un des outils méthodologiques du commentaire et de l'accès à la pensée philosophique.

Puis, après avoir publié quelques traductions, le hasard a voulu que je sois sollicité pour intervenir sur la traduction. Parallèlement, j'enseignais la philosophie à une époque où il y avait une explosion méthodologique de la linguistique. J'ai le privilège d'avoir été l'élève de Gaston Bachelard, de Jean-François Lyotard et de Jacques Derrida, de Raymond Aron et de Claude Lévi-Strauss, et surtout de Paul Ricœur, entre autres penseurs. Dans leur pensée, il était beaucoup question de linguistique. En tant que philosophe d'abord, j'ai été vers la linguistique par en haut; et en tant que germaniste, par en bas, c'est-à-dire que je me suis intéressé à la grammaire de l'allemand et à la structure de sa syntaxe. À la fois: à la linguistique descriptive et à la pensée linguistique, notamment celle de Saussure. C'est ainsi que je me suis trouvé être sollicité à intervenir dans ce domaine; et le sujet qui m’a bien sûr spontanément intéressé, c'était la traduction. En 1972, Jean Dubois m'a confié la direction d'un numéro sur la traduction de la revue Langages, revue prestigieuse de linguistique. 
Du coup, j'écrivais et je réfléchissais sur la traduction en elle-même, indépendamment d'un rapport direct à la philosophie, qui avait été mon entrée en traduction. Ce numéro de revue a marqué le début d'une bifurcation faisant que j'avais deux métiers intellectuels, qui n'étaient pas opposés mais complémentaires - à savoir la philosophie, que j'enseignais à l'Université de Paris-X-Nanterre, et la traduction envisagée de façon autonome par rapport à la philosophie. En tant que philosophe, je me suis intéressé à la traduction, non seulement en traduisant Habermas, mais également Adorno, Nietzsche, Kant et d'autres philosophes. Par la suite, j'ai pensé réunir, ou plutôt approfondir et synthétiser, différentes études en publiant mon livre Traduire: théorèmes pour la traduction. En fait, j'ai publié mes théorèmes pour la traduction après avoir traduit une dizaine de livres. Tout philosophe que j'étais (et que je suis encore), mon idée toutefois n'était pas d'intervenir sur un champ social comme la traduction a priori: tout ce que j'ai pu écrire, même si je lui donne la forme conceptuelle de la philosophie et de la linguistique, reste éminemment gagé sur la pratique. S'agissant de traduction, je ne cesse de me référer à la pratique, dans chaque phrase que j'écris.

\section{- En somme, vous avez découvert que la traduction était un opérateur omni- présent dans votre réflexion...}

On pourrait dire, en paraphrasant Clausewitz, que si «la guerre est la continuation de la politique par d'autres moyens», le texte-cible est la continuation du texte-source par d'autres moyens. Finalement, l'idée d'une dialectique de l'«interpénétration» du Même et de l'Autre dans la traduction est un topos extrêmement concret, presque banal, qui fournit un modèle de réflexion intéressant et important. D’année en année, la traduction a pris une importance de plus en plus grande pour moi. Au lieu d'être un simple outil au service de la philosophie, c'est devenu un travail, un métier et une écriture seconde autonomes, puis un objet de réflexion et de recherche, et enfin un paradigme anthropologique et philosophique. De ce fait, j'ai été invité à droite et à gauche et j'avais coutume de dire: «la traduction, c'est mon agence de voyages!», ce qui pouvait sembler un peu méprisant, mais ce n'était pas du tout le cas, c'était de l'auto-dérision. Comme disait Pasteur (après Bacon), « un peu de science éloigne de Dieu, beaucoup y ramène» : eh bien! la traduction m'a ramené à la philosophie dont, dans un premier temps, elle m'avait éloignée. Tout cela va dans le sens de ce que j'ai appelé, de façon peut-être un peu ambitieuse, une anthropologie interdisciplinaire de la traduction.

\section{- Quels seraient, d'après vous, les fondements épistémologiques de la traduc- tologie comme discipline?}

C'est une question difficile. Certes, on pourrait penser que je suis assez bien placé pour y répondre, puisque j'ai beaucoup travaillé en traductologie et que j'ai également enseigné la philosophie, notamment l'épistémologie des sciences humaines. Peut-on parler de fondements épistémologiques? Je serais un peu embarrassé pour répondre à cette question et je formulerai plutôt les choses ainsi: quelles sont les questions épistémologiques touchant la traductologie? D’abord: la traductologie est une praxéologie, c'est-à-dire une discipline, un savoir ou une «science» qui prend pour objet une pratique. Concernant ses fondements épistémologiques, cela supposerait qu'elle 
prenne appui sur des sciences constituées ou qu'elle ait un organon de principes définis au préalable. Ce qui m’a toujours guidé, quoique philosophe par ailleurs, c'est un très grand pragmatisme en cette affaire. Si fondements épistémologiques il devait y avoir, certains pourraient être tentés d'aller les chercher du côté de la linguistique, par exemple. En effet, pendant plusieurs décennies, la traductologie a été conçue comme une sorte d'appendice ou de province extérieure de la linguistique et des sciences du langage, c'est-à-dire de la dite Linguistique Appliquée. Bien sûr, il ne s'agit pas seulement de cela. Ma première réponse à votre question des fondements épistémologiques de la traductologie, ce serait paradoxalement de dire que je suis bien embarrassé pour y répondre dans la mesure où je ne vois pas qu'il y ait des fondements épistémologiques - ni là, ni ailleurs au demeurant...

Par ailleurs, j'aurais tendance à dire que la traductologie est une praxéologie qui ne s'autorise que de son rapport à la pratique, et non pas dans d'une discipline préalable ou dans de principes théoriques préalables. Cela dit, force est de constater que la traductologie est une science jeune et même "frêle», en sorte qu'il lui faut prendre appui sur des disciplines traditionnelles, plus assises et plus solides. Les traductologues viennent souvent d'autres disciplines. Ce sont souvent des linguistes, mais en Europe, ce sont moins des linguistes de linguistique générale (que j’appelle des «linguisticiens ») que des anglicistes, des francisants, des germanistes, des hispanistes..., qui ont travaillé spécifiquement une langue, une littérature et une civilisation particulières (que j’appelle des «languistes»).

\section{- Certains viennent aussi de littérature comparée.}

Oui, bien sûr! Beaucoup viennent aussi de la littérature comparée! comme Sherry Simon, Jean-Yves Masson et bien d'autres. Par contre, nous sommes très peu à venir de la philosophie. Comme je l'ai dit, je suis venu à la traductologie d'abord par l'expérience de la traduction. À la lumière de cette expérience, ma formation interdisciplinaire en linguistique et germanistique, d'un côté, et en philosophie de l'autre, m’ont amené à réfléchir sur les processus de traduction et à élaborer ma propre traductologie.

On pourra s'étonner que les fondements épistémologiques de la traductologie soient extrêmement friables, puisque c'est une praxéologie et qu'elle s'appuie sur des disciplines déjà préexistantes, mais en prenant de grandes libertés par rapport à elles. À vrai dire, ni la linguistique ni la littérature comparée n'apportent de fondements épistémologiques. Ces disciplines apportent des éléments interdisciplinaires de méthodologie, de conceptualisation, de terminologie. Au fond, on aurait là une discipline sans fondements épistémologiques à proprement parler et qui, corollairement, est sans cesse en train de redéfinir son objet. D’ailleurs, c'est peut-être plus généralement le cas de beaucoup d'approches et de disciplines en sciences humaines dans la mesure où, comme on ne parvient pas à constituer une théorie scientifique de l'objet, on est amené à redéfinir constamment le champ de recherche dont on traite. J'ai beaucoup insisté sur le fait que ce qui m’intéressait, c'était la traduction au sens dynamique, en tant qu'activité traduisante, c'est-à-dire le traduire. Tel est l'objet de la traductologie à mes yeux, alors que beaucoup s'intéressent à la traduction au sens statique d'un résultat. À ce propos, je dirai le traduit, comme on parle d'un «produit». D'où des études comparatives ou contrastives entre le texte original et sa traduction (ou ses traductions): cela correspond à ce que j'appelle la traductologie descriptive. 


\section{- Vous faites référence à la typologie à quatre termes que vous avez proposée : pourriez vous rappeler ici votre modèle?}

En effet, j'ai proposé une typologie à quatre termes définissant quatre âges de la traductologie. Il y aurait eu d'abord la traductologie prescriptive ou normative, où je regroupais des considérations plus ou moins philosophiques, des aperçus littéraires et des conseils pratiques et qui, rétrospectivement, m'apparaît un peu comme une catégorie «fourre-tout». En fait, c'est à mes yeux la traductologie d'avant-hier. Puis vient un deuxième âge, une deuxième catégorie, la traductologie descriptive, d'obédience linguistique, qui correspond à la majorité de ce qui se publie et qui apparaît comme un prolongement appliquant les concepts de la linguistique. À l'endroit de la traductologie descriptive, je formulerai plusieurs critiques: surtout, elle s'intéresse aux traductions déjà faites et elle s'en tient donc à des comparaisons entre le texte original (To) et sa traduction (Tt), voire plusieurs de ses traductions ( $\mathrm{Tt}, \mathrm{Tt}$ ', $\mathrm{Tt}$ », $\mathrm{Tt}$ »....). L'un des instruments de travail est constitué par des corpus de bi-textes (To $+\mathrm{Tt}$ ). Je reformulerai plus clairement ma critique en disant que je ne m'intéresse pas tant à la façon dont un autre a traduit un texte hier qu'à la façon dont je vais traduire un texte aujourd'hui. Il me semble que la critique traductologique, du moins telle que je l'entends, et la pratique devraient conduire à poser cette question-là et à y apporter des éléments de réponse, sinon la réponse définitive. Je dis bien: des éléments de réponse, dans la mesure où en traductologie, et d'une façon générale en sciences humaines, nous ne sommes pas en mesure de fournir une théorie globale qui serait expérimentalement validée. Le mieux que nous puissions espérer, c'est d'apporter des éléments de réponse partiels: partiels, étant donné que ce ne sont que des éléments, éventuellement aussi contradictoires. Ainsi certains vont défendre telle position, d'autres la position opposée, sans que l'on puisse dire de façon aussi claire qu'en sciences exactes que les uns ont tort et les autres raison.

Le travail de la traduction comporte des éléments exaltants: quand on traduit un texte qu'on aime, qu'il nous vient des trouvailles de traduction... Mais c'est aussi un travail fastidieux au jour le jour. Par conséquent, il y a une sorte d'escapisme théorique chez certains traductologues, comme si la théorie était une façon de prendre congé de la pratique qui, à certains égards, est un peu pesante, comme si c'était pour rehausser un peu le caractère besogneux de la traduction. D'ailleurs, on a tendance à l'oublier de nos jours: même pour un écrivain, écrire, c'est aussi un travail laborieux...

\section{- Freud parle de "Durcharbeitung» (perlaboration), un concept que reprend Paul Ricœur dans ses écrits sur la traduction.}

J'emploie moi-même très souvent le terme de travail - en un sens plein, que je me plais à appeler obstétrico-psychanalytique. Cela rejoint la Durcharbeitung chez Freud. De même, on dira d'une femme sur le point d'accoucher qu'elle est «en travail» ou que, dans une maternité, elle entre en «salle de travail». Le sens obstétrical rencontre le sens psychanalytique dans des formules comme le «travail du deuil» ou le «travail du rêve». L'idée est que ce travail-là, en somme, c'est premièrement un travail sur soi, deuxièmement un travail qui prend du temps, troisièmement qui est douloureux ou pénible, et quatrièmement, qui débouche sur une sorte d'accouchement. En obstétrique, c'est un vrai accouchement; mais l'écriture en traduction "accouche» de nos enfants de papier, faute de faire des enfants de chair. 
Il y a dans le domaine de la traductologie une certaine incertitude et on entend des discours contradictoires, multiples et parfois incohérents. Les mêmes prétendent et avancent des choses tout à fait incompatibles les unes par rapport aux autres, sans que cela semble les gêner beaucoup! Il y a à cela, me semble-t-il, deux raisons: une bonne et une mauvaise. La bonne, c'est qu'étant donné qu'il s'agit d'une pratique, il faut la prendre là où elle est; et, par définition, elle n'est pas forcément conforme à nos exigences de "propreté» théorique. Il est normal qu'il y ait alors des contradictions. Sauf qu'une contradiction, c'est quand même un état immature de la réflexion. Il faut se donner, par après, la peine d'être assez précis pour voir où il y a des complexités dans le réel qui amènent à tenir des discours eux-mêmes complexes, et qui peuvent superficiellement sembler contradictoires dans la mesure où les conditions d'application respectives n'ont pas été précisées assez explicitement. Même si la réalité est complexe, notre discours ne doit pas être compliqué! Ce qui est un manque théorique, c'est de prétendre de façon péremptoire, dogmatique ou générale des choses qui sont incompatibles entre elles ou, pire! qui sont contraires à la réalité. Cela dit, la pratique amène à formuler des discours nuancés et à prendre, par rapport à tel ou tel problème, des positions qui peuvent être opposées les unes par rapport aux autres, compte tenu des différents domaines respectifs d'application qui ne sont pas les mêmes.

\section{- En somme: iriez-vous jusqu'à dire qu'il n'y aurait pas vraiment d'incohé- rence dans certains discours?}

Il y a aussi une mauvaise raison à l'incohérence et à la confusion de certains discours: c'est la désinvolture intellectuelle de certains, le manque de sérieux, quand ce n'est pas un déficit de rationalité ! sans parler de la mauvaise foi... Ce qui est vrai, c'est que les fondements épistémologiques dans certains cas sont introuvables, et pour cause! Les fondements épistémologiques de la traductologie, comme de beaucoup de sciences humaines, tout particulièrement des praxéologies, reposent sur des sables mouvants. On doit donc sans arrêt faire un travail de reformulation des fondements supposés, des hypothèses portant sur les fondements possibles du travail qu'on est en train de faire. La difficulté est que si les fondements ne sont pas clairs, ce qui doit être clair, c'est le discours que l'on tient sur le fait que ces fondements ne sont pas clairs. On ne saurait être plus clair!

On a un problème analogue en politique et en philosophie, à savoir qu'on n'arrive pas à convaincre tel ou tel d'adopter les mêmes positions politiques ou philosophiques que les siennes par la raison seule. Mais cela n'exclut pas qu'il y ait de la rationalité. Les dogmatiques s'imaginent que leur position est rationnelle et que celle des autres ne l'est pas. Les gens raisonnables, qui sont très peu nombreux, seraient fondés à penser qu'il est assez rare qu'il y ait de véritables erreurs de raisonnement: dans le meilleur des cas, l'argumentation est rationnelle, même si beaucoup de théoriciens en traduction, comme dans d'autres domaines, ne sont pas gênés par les contradictions de leur discours. L'erreur vient d'ailleurs. Alors, quel est le problème? L'erreur vient des présupposés et des hypothèses qui sont différents et sont restés implicites. Or, si les présupposés sont différents et si le raisonnement est cohérent, il est bien naturel qu'on aboutisse à des conséquences différentes et à des points de vue opposés. Qu'on ne puisse pas convaincre l'autre, c'est dès lors bien naturel, puisque ce n'est pas 
une faute de raisonnement qu'on pourrait démontrer et dont on pourrait convenir. L'origine du problème remonte aux présupposés implicites et «décisionnels» (cf. Habermas). Paradoxalement, ce qu'on ne peut pas démontrer, c'est ce à quoi on tient le plus. Tout au plus devrait-on pouvoir arriver à un méta-consensus sur les présupposés sous-jacents du dissensus. En traductologie, c'est moins évident; c'est pourquoi j'ai pris l'exemple de la philosophie et de la politique. Ma position méta-politique s'inspire de la philosophie politique de Habermas, mais aussi de l'axiomatique d'un Hilbert. Ainsi, dans le meilleur des cas, une traductologie cohérente et exigeante serait comme toute science humaine, toute philosophie ou toute position politique: elle procède de présupposés qui ne sont pas démontrés et qu'il faut seulement expliciter. Cela implique qu'il faille d'abord en prendre conscience, dans la mesure où ils ne sont pas de l'ordre de l'évidence et qu'il est possible d'en avoir d'autres. Je n'en veux pour preuve que les polémiques opposant «sourciers» et «ciblistes».

\section{- S'agissant des sciences humaines et de la traductologie, quelle serait la ques- tion essentielle qu'il faudrait se poser?}

La question est celle-ci: est-ce que mes présupposés - ou les vôtres - amènent à développer un discours plus fécond, plus fidèle à la pratique, plus productif que celui de tel ou tel autre? À moins que vos présupposés soient productifs dans tel ou tel domaine de la traduction, alors que ce seraient mes présupposés qui permettraient de dégager un discours théorique plus éclairant, plus efficace dans la même discipline et dans d'autres domaines. Je pense qu'une part importante du caractère parfois un peu polémique et exalté de certaines discussions en traductologie réside dans ce déficit d'auto-critique épistémologique (ou «méta-traductologique»), qui fait l'impasse sur le métalangage logique, philosophique, épistémologique que je viens d'expliciter et qui sous-tend les sciences humaines en général. Il arrive souvent, comme ici, que la traductologie soit significative des sciences humaines. En outre, il vient s'ajouter à cela la surdétermination d'interférences idéologiques.

La plupart du temps, les positions qu'on défend à partir de présupposés qu'on ne peut pas démontrer, mais auxquels on tient beaucoup paradoxalement, représentent le noyau non rationnel auquel on tient le plus. D'ailleurs, c'est naturel après tout, car la raison est à tout le monde. On n'a pas lieu d'y tenir, comme à ses propres présupposés. En revanche, nos présupposés, nos amours, nos convictions religieuses ou politiques, c'est vraiment à nous! Donc, il est normal qu'on y tienne d'une certaine façon comme à sa propre identité. On y tient aussi parce qu'éventuellement on les sent fragiles, puisque d'autres peuvent avoir différents présupposés. Il faut donc les défendre, croit-on. Dans une de mes traductions de Habermas, j'ai parlé de «résidus décisionnels » pour traduire une expression allemande. Il s'agissait des résidus décisionnels qui sont au principe de l'argumentation. La raison, on n'a pas besoin de la défendre, pas même contre les sots qui, en principe, ne représentent pas un adversaire dangereux (sic). D’une façon générale, la question des fondements épistémologiques est sans doute un point aveugle de la réflexion traductologique.

- Vos remarques sur les présupposés rappellent ce que dit Hans-Georg Gadamer. La seule objectivité qui puisse exister en sciences humaines, selon lui, est de prendre conscience de ses propres préjugés, et les préjugés valables 


\section{sont ceux qui s'avèrent les plus féconds. N'auriez-vous pas là une position qui rejoindrait celle de l'herméneutique philosophique?}

Oui, bien sûr: j’ai pratiqué Gadamer, mais également beaucoup la Théorie critique de l'École de Francfort, notamment dans sa deuxième génération incarnée par Jürgen Habermas. On constate que tous ces penseurs sont souvent plus proches les uns des autres qu'on aurait cru. En ce qui me concerne, j'ai d'abord travaillé sur Habermas et ensuite sur Gadamer, et j'ai vu qu'une partie des arguments que Gadamer développe avec beaucoup plus de précision, et dans des domaines où cela convient mieux, étaient déjà présents chez Habermas. En effet, dans la controverse intellectuelle, on est souvent amené à reprendre les points forts de l'adversaire en essayant de les intégrer à sa propre position. C'est cela le bon usage de la polémique dans nos domaines, dans la mesure où la discussion permet à chacun de renforcer sa position au sens où on en élimine les points faibles en reprenant à l'«adversaire» des éléments de vérité. D'une certaine façon, la polémique est un rapprochement lorsqu'elle est honnête et sérieuse.

\section{- Cela a été le cas pour Gadamer et Habermas...}

Oui, c'était vraiment le cas et même - selon une formule de Haroldo de Campos, que j'emploie me concernant - on aurait une sorte de "cannibalisme théorique ", à savoir une synthèse sélective. Lorsque je suis en opposition avec quelqu'un sur le plan intellectuel, j'essaie toujours de «manger» ce qu'il apporte, de l'intégrer, de le phagocyter. De mon point de vue, je n'ai pas à combattre une idée, à tort ou à raison. J'ai toujours tendance à préférer intégrer les idées, à les manger; ce serait mon modèle de la discussion intellectuelle. Avant la systématisation de la tradition herméneutique par Gadamer et la part qu'a pu en reprendre Habermas, je dirai que d'une certaine façon c'est le bon sens même qui nous le suggérait. Comme souvent, les grandes pensées philosophiques nous rappellent à des évidences oubliées, à des intuitions ensevelies, qui sont comme une sorte d'Atlantide du bon sens qu'on serait amené à faire réémerger. Cela rejoint la Destruktion chez Heidegger, qui a été le Maître de Gadamer.

En ce qui concerne la traductologie: outre qu'elle se situe en aval des disciplinesmères précédemment évoquées - que sont la linguistique, l'étude des langues étrangères, la littérature comparée, la psychologie ou la philosophie - sa base méthodologique, ses fondements épistémologiques et ses concepts-clefs seraient définis dans ce que j'ai appelé un «triangle interdisciplinaire».

\section{- Comment définissez-vous ce "triangle interdisciplinaire» de la traducto-} logie?

Je le définis de la façon suivante: un triangle dessiné par les sciences du langage, à savoir la linguistique, d'une part, puis un sommet plus haut qui serait la philosophie, et puis un sommet plus bas qui serait la psychologie. Les sciences du langage et la linguistique sont directement concernées parce qu'il est question de langues, de mots et de textes, etc. L'acquis conceptuel ou réflexif de la théorie linguistique et des sciences du langage a lieu d'être mis à contribution pour cette réflexion sur les objets langagiers desquels nous partons pour le texte-source et sur lesquels nous débouchons pour ce qui est du texte-cible. Mais, encore une fois, la traductologie ne peut pas se contenter d'être une province ou un appendice de la linguistique et des sciences du 
langage. On ne traduit pas des langues, mais des textes; et même on ne traduit pas tant des textes que le sens et les effets de discours dont ils sont porteurs.

Si j'introduis la philosophie, c'est à la fois parce que c'est l'un des horizons intellectuels dont je viens de parler à propos de Gadamer et de Habermas, mais aussi parce que la philosophie permet précisément d'échapper à l'enfermement méthodologique. À la différence des sciences humaines par exemple, la philosophie est libre de sa production intellectuelle; c'est même éventuellement le reproche qu'on peut lui faire. Elle va dans tous les sens; et l'un des griefs que l'on fait aux philosophes est qu': «ils ne sont même pas d'accord entre eux»! ce qui est vrai. Il y a une grande productivité et une grande inventivité, souvent tout à fait étonnantes. C'est aussi fascinant que l'étrangeté débusquée par l'ethnologie quand, par exemple, on s'interroge sur la pensée des philosophes dits cartésiens (qui ont en commun d'avoir critiqué Descartes) comme Leibnitz, Berkeley, Spinoza et Malebranche. Descartes lui-même, est à l'origine de ces analyses concernant le rapport entre la pensée et l'étendue, le rapport à Dieu, etc. On a parfois l'impression que ce monde de la philosophie classique est un tout autre monde que celui dans lequel nous vivons.

Il m'est arrivé d'utiliser la formule suivante: «les sciences humaines imitent les sciences exactes». J'aime cette métaphore platonicienne. Toutefois, ces sciences humaines ne peuvent arriver à la même rigueur méthodologique ou théorique, voire à la même rigueur quantitative que les sciences exactes - sauf dans des secteurs limités, comme la psychologie expérimentale ou la démographie - mais elles s'en inspirent. Faute d'appliquer la méthode expérimentale et de mettre en œuvre une formalisation logico-mathématique de leurs énoncés, les sciences humaines visent à un discours rationnel et à une validation par l'expérience. Mais cette validation par l'expérience qu'elles recherchent est plus limitée qu'en sciences exactes: elles doivent se contenter de ce que j'appelle une plausibilisation empirique de leur discours. Cependant, cela implique déjà une certaine rigueur conceptuelle, qui s'interdit de faire fond sur des intuitions qu'on n'a pas préalablement intégrées dans un système conceptuel et qui sont encore en attente de plausibilisation empirique. C'est la raison pour laquelle je tiens à souligner que la philosophie, elle, échappe à l'enfermement méthodologique des sciences humaines; a fortiori en est-il de même de la littérature. La formule que j'emploie, par analogie à une formule de Freud sur la psychanalyse sauvage, est que la philosophie nous permet de développer une sémantique sauvage, c'est-à-dire une sémantique qui n'est pas astreinte à la scolastique conceptuelle et méthodologique exigée par les sciences du langage.

\section{- Vous dites qu'il y a un clivage au sein des sciences humaines?}

Cette opposition apparaît clairement en psychologie, entre la psychologie expérimentale et la psychologie clinique. Les cliniciens décrivent de façon très circonstanciée un vécu pathologique, une trajectoire de vie, une expérience infantile ou enfantine (infantile serait peut-être trop directement psychanalytique), un parcours névrotique, etc. Il font de la "clinique», c'est-à-dire un examen de ce que vit le sujet. J'userai volontiers ici du terme de "phénoménologie», comme on tend à l'employer maintenant, en un sens un peu différent de celui de l'héritage de Husserl et de Heidegger. En somme, on trouve d'un côté le travail des cliniciens, qui étudient de façon non scientifique quelque chose de réel. De l'autre côté, on trouve les expérimentalistes qui veulent mettre en place (à juste titre) la méthode expérimentale telle qu'elle est à 
l'œuvre dans les sciences, mais qui du même coup sont forcés d'éliminer tout un ensemble de facteurs afin de parvenir à formaliser et à quantifier leur objet. Voilà donc d'un côté les expérimentalistes qui étudient de façon scientifique un objet non réel et, de l'autre, les cliniciens qui examinent de façon non scientifique un objet réel.

\section{- N'y aurait-il pas là une alternative épistémologique dont on ne sort que difficilement et qui représenterait le cadre conceptuel des sciences humaines?}

Je systématise pour les besoins de l'argumentation ces deux extrêmes, qui nous donnent une polarité épistémologique. Ce couple expérimentalistes/cliniciens, qui nous vient de la psychologie, on le retrouve mutatis mutandis dans toutes les sciences humaines. En traductologie, il y a une opposition entre les théoriciens d'une discipline de recherche et les théoriciens d'une pratique. Dans les deux cas, on théorise. Mais, dans l'un des cas, on théorise par rapport à la volonté de constituer une discipline avec ses exigences épistémologiques spécifiques. Dans l'autre, celui de la praxéologie, les théoriciens s'efforcent de théoriser la pratique dans toute sa complexité, en n'en rabattant de l'exigence théorique, comme les cliniciens peuvent le faire en prenant tout le vécu du patient, en consentant à une théorie beaucoup plus souple. D'ailleurs, parmi les cliniciens pratiquant actuellement la psychothérapie, les meilleurs ne sont souvent pas les tenants d'une obédience théorique stricte: freudiens, lacaniens, cognitivistes ou autres. Ils n'hésitent pas à aller puiser du côté des psychanalystes, des comportementalistes, de la psychologie génétique de Piaget, voire de la psychologie transgénérationelle, de l'analyse transactionnelle etc. Par analogie, j’ai employé la formule suivante pour prendre à mon compte ce changement en théorie de la traduction: il y a un "tournant œecuménique» en traductologie. En France, il y a souvent une base freudienne importante, mais qui n'exclut pas des emprunts aux thérapies comportementalistes; alors que c'est totalement contradictoire sur le plan théorique. L'important, lorsqu'on a un patient qui souffre, c'est de l'aider à moins souffrir et à se tirer d'affaire. C'est d'ailleurs ce que disait Freud lui-même. Ce que voulait Freud - car c'était d'abord un médecin avant d'être un théoricien, un philosophe et un écrivain - c'est que ses patients aillent moins mal. Comment faire pour qu'ils aient moins mal? Le problème du clinicien est de prendre en compte le réel avec sa complexité; et puis la théorie, elle suit comme elle peut... Éventuellement, on assume une théorie de bric et de broc.

\section{- Pourriez-vous développer le parallèle que vous avez évoqué entre psychothé- rapie et traductologie?}

Si j'ai parlé de ces problèmes, c'est parce qu'ils m'intéressent à titre personnel, et que la psychothérapie est une praxéologie, comme la traductologie. Dans un cas, il s'agit de gérer la pratique de la traduction, et dans l'autre, de gérer la pratique d'un soignant par rapport à un problème, celui de la souffrance d'un patient. Dans le premier cas, c'est un texte avec ses difficultés dont on a du mal à résoudre. Donc, je mets en rapport ces deux praxéologies. Il est intéressant que les psychothérapeutes aient conscience qu'il y a plus d'urgence à diminuer la souffrance d'un patient, que de supprimer un contresens théorique. En cela, les psychothérapeutes ont sans doute été en avance par rapport à bien des traductologues! 
Beaucoup emploient des mots comme «cognitif» ou «comportementaliste», en les mettant dans le même sac. Le comportementaliste serait l'extension d'une vision de type réflexologique, où il s'agit de déconditionner un patient de ses blocages douloureux. D'une certaine façon, ce serait le contraire du cognitif. La cognition, en principe, se passe au niveau intellectuel. Toutefois, à un certain niveau, les choses se rejoignent, puisqu'on va en appeler à la connaissance et à la réflexion pour aider le patient, en tablant sur la conscience (au moins partielle) qu'il a de son état et sur sa volonté de «s'en sortir», dans un dialogue pour introduire des mesures volontaristes prudentes et progressives d'auto-déconditionnement sous le contrôle du thérapeute. Il faudrait aussi rappeler ici ce qu'on peut emprunter à la psychanalyse culturelle de Jung ou à la psychologie de l'ego d'un Adler. Dans ce contexte, je ne peux pas ne pas citer une personnalité captivante comme Marie-Louise von Franz, qui se situe dans la mouvance de Jung.

\section{- Mais alors, quel statut revient-il encore à la théorie?}

En traductologie, vous avez encore des appartenances théoriques. Elles sont affichées ou non, explicites ou implicites, conscientes ou inconscientes; mais il y a bel et bien des appartenances théoriques. Or, le travail à faire, c'est de mettre en évidence les théories implicites ou explicites, et de s'engager dans ce travail de "cannibalisme» dont je parlais tout à l'heure. Il faut le faire à la fois au niveau proprement théorique, mais surtout - et là on rejoint ce que nous disions du concept de "préjugé» (VorUrteil) à propos de Gadamer - par rapport à ce que telle ou telle théorie permet au niveau de la pratique, quitte à ce qu'il y ait d'autres théories et que règne une certaine incohérence. Dans un article de jeunesse, j’ai plaidé pour une gadgétisation de la théorie, pour l'incohérence théorique. La formule que j'ai souvent employée est de dire que ma traductologie est «une rhapsodie de théorèmes disjoints affrontés à la tourmente de la pratique». Rhapsodie est à prendre ici au sens de Kant: lorsqu'il emploie le terme de rhapsodie, c'est en un sens péjoratif et critique; cela veut dire qu'on n'a pas affaire à l'architectonique d'une philosophie cohérente.

Dans le contexte qui nous occupe ici, la première chose à observer, c'est l'attention à la pratique. C'est le sens que prend le mot phénoménologie dans le langage non spécifiquement philosophique. C'est l'idée que je fais l'effort de mettre entre parenthèses (et non pas d'oublier) mes a priori théoriques, pour être attentif à ce qui advient, au ressenti du rapport à l'autre dans la psychothérapie ou la dynamique de groupe. Je reprends ainsi le terme en traductologie: être attentif au ressenti langagier de l'énoncé que j'ai à traduire (To); et, dans un deuxième temps, au ressenti langagier de l'énoncé que j'ai écrit moi-même (Tt) et qui fait sens par lui-même. Mon «théorème du gâteau au four " répond à cette expérience: on pense que l'on a voulu dire cela, et puis il sort autre chose. Ou plutôt: il me vient une phrase où j'ai voulu signifier telle ou telle chose, et quand je la relis, elle fait sens par elle-même. Comme un gâteau qui, une fois sorti du four, est très différent de la pâte que l'on avait mise dans le moule avant de l'enfourner.

\section{- Ce théorème du gâteau au four fait, donc, partie de vos cinq théorèmes culinaires...}

Je reformulerai ce théorème du gâteau au four de façon plus savante en disant que c'est la dialectique du décalage entre le vouloir dire psychologique initial du traducteur 
dans sa tête et le vouloir-dire sémantique terminal de son texte sur le papier. Très souvent, quand nous relisons nos textes, ils font sens indépendamment de nous. C'est ce que j'appelle l'Esthétique de la traduction, l'attention portée au ressenti langagier de mes propres énoncés et de l'énoncé source. Dans le cas de la théorie interprétative de la traduction (TIT), pour Danica Seleskovitch et Marianne Lederer, il convient de traduire le "vouloir dire». Si j'ai proposé mon théorème du décalage entre les deux sens de "vouloir dire», c'est que j'ai repris ce concept, en relevant qu'il est flou. Cela dit, ce flou est d'une certaine façon fécond. C'est pourquoi j'ai distingué le vouloir dire psychologique ou mental de l'auteur (sans trait d'union) du vouloir-dire sémantique du texte (avec trait d'union). Ce flou est porteur parce que, dans un premier temps, on ne peut guère vraiment distinguer le vouloir-dire sémantique du texte source et le supposé vouloir dire de son auteur. Quant à moi, j’ai souvent employé la formule suivante: «on ne traduit pas ce qui est écrit, on traduit ce qu'on pense qu'a pu penser celui qui a écrit ce qu'il a écrit quand il l'a écrit». On ne peut pas faire plus simple!

Encore une remarque à propos des fondements épistémologiques de la traductologie et du triangle disciplinaire linguistique, philosophie et psychologie. Pour ce qui est de la psychologie, il faut partir de «ce qui se passe dans la tête des traducteurs » (H. P. Krings), et notamment du traducteur que je suis moi-même quand je traduis. Aussi suis-je attentif à ce que je pense du texte, aux difficultés que j'ai à traiter. C'est pourquoi une part importante de la traductologie consiste à ce que je me plais à appeler une sorte de traducto-thérapie. Avec mes étudiants, j’assure des séminaires de «clinique traducto-thérapeutique».

\section{- Comment se passent ces séminaires de «clinique traducto-thérapeutique»?}

Ce n'est pas du genre: «Allongez-vous, parlez-moi de vous, et de vos difficultés (de traduction), dites-moi comment vous vivez le texte...» Le point important, c'est l'auto-analyse, l'attention aux erreurs qu'on a pu faire, aux fausses pistes sur lesquelles on est tenté de partir. J'entends aider mes étudiants à opérer ce que j'appelle un "auto-accouchement de leur idiosyncrasie de traducteur». Cette maïeutique traductologique aide à faire prendre conscience de ses points forts et de ses points faibles, pour jouer de ses points forts, et se garder de ses points faibles. À titre personnel, je ne vais pas renoncer à certaines de mes manies stylistiques, car il y a des mots et des tournures que j'aime bien. J'emploie souvent "d'emblée», une expression qu'affectionnait Bergson, ou "du coup»; mes étudiants le savent et s'en amusent. Comme on sait, je traduis le plus souvent de l'allemand. Etant donné que les phrases allemandes sont fréquemment assez longues, la plupart des traducteurs les coupent. En ce qui me concerne, je mets un point d'honneur à ne pas les couper: c'est une décision personnelle, qui relève de mon idiosyncrasie de traducteur. Dans l'institution pédagogique, «nous avons un fallacieux idéal de la phrase courte et paratactique» (comme je l'indique dans mes Théorèmes). En l'occurrence, cela relève du talent de l'écriture, et c'est un choix. J'essaye de maintenir les phrases longues parce que maîtriser la langue, selon la bonne rhétorique cicéronienne, c'est maîtriser la période et la sentence. La période, qui est longue et bien construite, a de l'ampleur; et la sentence, qui claque à la fin (ou au début), énonce avec vigueur et fait formule.

Il arrive que les traducteurs écrivent mieux que certains de ceux qu'il leur arrive de devoir traduire. Souvent dans les institutions internationales, on a à traduire (notamment à partir de l'anglais) des textes rédigés par des personnes qui ne savent 
pas forcément l'anglais: ils écrivent des Rapports qui sont «cochonnés» du point de vue de l'écriture et parfois incohérents quant au contenu. Or c'est au traducteur qu'on imputera la mauvaise qualité du texte final! Ce n'est pas le moindre scandale que ce soit au traducteur de sauver le texte en dernière instance. D’abord, il doit l'écrire en bon français, alors que le travail en «anglais»-source peut avoir été mal fait; et même si c'est incohérent, il lui faut introduire de la cohérence. Heureusement: cette cohérence, on finit par la trouver le plus souvent, quitte à devoir émettre des hypothèses. On se demande ce que l'auteur a bien voulu dire. Dans de telles conjonctures, je dis à mes étudiants: «regardez ce que l'auteur a dit avant et ce qu'il a dit après». En général, on arrive à un texte cohérent avec une marge d'incertitude, certes, mais avec une grande plausibilité. Ce qui est intéressant, c'est justement ce travail psychologique. Ainsi le traducteur est-il condamné à la créativité, contrairement à ce que les gens extérieurs au milieu de la traduction s'imaginent. Pour beaucoup, le traducteur, c'est l'homme invisible!

Pour en revenir à notre triangle interdisciplinaire: la psychologie, c'est donc une attention portée à ce qui se passe dans la tête du traducteur; et puis, c'est aussi l'autoaccouchement de son idiosyncrasie de traducteur. Au reste, la psychanalyse est aussi une philosophie: les concepts de la psychanalyse contribuent également à ce que j'ai appelé une sémantique sauvage. Freud lui-même fait la comparaison entre traduction et psychanalyse, car la psychanalyse fait passer un contenu latent, comme si c'était un texte-source, au niveau d'un langage patent, comme si c'en était la traduction. La psychanalyse est une interprétation, une herméneutique; du reste, le livre de Ricœur sur Freud s'intitule De l'interprétation; ce qui est aussi une allusion à la traduction et au De Interpretatione d'Aristote.

\section{- Souscririez-vous à l'idée de Ricœur, développée notamment dans Le conflit des interprétations, que la psychanalyse serait sinon une discipline philoso- phique, du moins une discipline pour le philosophe?}

Oui, tout à fait. Ricœur parle aussi de l'herméneutique des profondeurs (Tiefenhermeneutik): c'est un concept qu'on retrouve aussi chez Habermas (dans l'un des derniers chapitres de Connaissance et intérêt) et qui remonte en principe à Jung. On retrouve la psychanalyse à plusieurs niveaux. Je dirai que la psychologie, qui constitue le troisième sommet (celui du bas) de mon triangle interdisciplinaire, est essentiellement orientée du côté de la psychologie de la personnalité, et donc de la psychanalyse, comme aussi de la psychologie générale et des théories traditionnelles qu'elles a engrangées. On a tendance actuellement à beaucoup trop privilégier la psychologie cognitive, parce qu'elle est censée être scientifique.

Mais je reviens à ce que je disais tout à l'heure. Je définissais une traductologie normative ou prescriptive comme étant la traductologie d'avant-hier, et je lui opposais une traductologie descriptive, qui est pour moi la traductologie d'hier et qui est essentiellement d'ordre linguistique. Je plaide pour ce que j'appelle une traductologie productive, qui s'intéresse non pas à la traduction déjà faite, mais au processus de traduction, d'où cette référence à la psychologie et à une sémantique sauvage. C'est à mes yeux la traductologie d'aujourd'hui. La question est de savoir comment je vais traduire. C'est tout le propos de mes Théorèmes.

Ce que j'écris à propos de la traduction est issu essentiellement de mon expérience de la pratique traduisante et, par ailleurs, c'est aussi une réflexion sur le statut 
de la discipline. Il faut réfléchir aux conditions de possibilité d'un discours traductologique, pour parler comme Kant. Paradoxalement, les trois instances que constituent la pratique traduisante, la théorie traductologique qu'on en induit et la méta-théorie épistémologique qui fait réflexion sur cette dernière, tendent à se rejoindre: elles sont directement pratiques.

Comme on l'a rappelé plus haut, j'ai distingué quatre âges de la traductologie; et les termes que j'ai choisis pour les désigner riment deux à deux : prescriptive/descriptive, productive/inductive. C'est pourquoi je me suis plu à qualifier cette typologie comme un quatrain traductologique, constitué d'un double homéotéleute... Au-delà d'une coquetterie terminologique et d'un jeu auto-ironique, il y a aussi le fait que notre esprit a besoin de mots pour penser. Il faut que les mots soient bien formés, y compris pour porter des idées abstraites (même si au départ les mots sont concrets et pour qu'ils permettent un maniement aisé). C'est pourquoi j’apporte le plus grand soin à mon design terminologique! Et je ne suis pas le seul. Une théologienne protestante comme Lytta Basset, pour bien marquer la nécessité psychologique de la différentiation, souligne que le fusionnel, la fusion, mène à la confusion. C'est une des surprenantes faiblesses de l'esprit humain qu'il se trouve être ainsi asservi aux idoles lexicales que sont les mots qu'il s'est créés. D’où l'étrange nécessité d'un travail stylistique, et même littéraire, de la pensée...

Encore quelques précisions touchant mon quatrain traductologique. Le quatrième âge, ce serait donc la traductologie inductive ou scientifique. Il s'agit d'une étude scientifique à mener sur ce qui se passe dans la tête, et même plus précisément dans le cerveau du traducteur. J'y ai vu la traductologie de demain; mais ce serait plutôt la traductologie d'après-demain, compte tenu de la complexité des phénomènes en cause.

Pour ce qui est de la traductologie productive, c'est du côté des psychologies de la personnalité que je me suis tourné, notamment de la psychanalyse, mais aussi du côté d'une psychologie plus traditionnelle, comme celle d'un Théodule Ribot ou comme la psychologie de la forme (Gestaltpsychologie), qui était restée finalement encore assez proche de la philosophie. Les éléments d'une sémantique sauvage vont me permettre, en cas de difficulté, d'analyser consciemment l'énoncé que j'ai à traiter et à hiérarchiser les contraintes. On ne peut pas tout dire, et la question que je me pose en tant que traducteur est celle-ci: «Qu'est-ce que j'accepte de perdre?»

À titre personnel, je dois beaucoup à mes études de psychologie, et notamment en psychopathologie clinique. J'ai suivi les cours de psychanalyse de Daniel Lagache. Ce qui est intéressant, c'est qu'on se dit «tout ça, c'est des trucs de fous!» Mais les fous, c'est nous! C'est une chose bien connue: quand on lit un Larousse médical, on croit qu'on a toutes les maladies, et toutes les névroses. Et pour cause! Parce que les névroses sont des fonctions normales qui sont déréglées au sens où elles prennent trop d'importance, pour compenser un manque ailleurs. C'est donc normal, d'une certaine façon, que nous ayons toutes les maladies - et pour la psychopathologie, c'est encore plus clair. Il y a quelques vingt ans, je participais à un colloque consacré à Henri Lefebvre, où j’avais fait une présentation qui avait été très bien reçue; et les gens me disaient en se moquant de moi affectueusement: "Jean-René, comment faistu? tu es formidable!» J'ai répondu: «mon talent ou mon génie, tiennent à une pathologie très chargée. Je parle comme un hystérique, je pense comme un paranoïaque et j'écris comme un obsessionnel...» La santé mentale, ce serait un cocktail de 
névroses équilibré: en réalité, l'équilibre psychologique, c'est d'avoir plusieurs névroses qui se contrebalancent. Le névrosé, c'est celui qui n'en a qu'une: un "très fou», par surabondance de folies, n'est pas vraiment fou; mais si on n'est qu'un peu fou, alors on est complètement fou! La théorie des névroses permet analogiquement de prendre conscience de notre idiosyncrasie de traducteur, puisque au bout du compte notre comportement normal est une variante névrotique.

La psychopathologie est aussi une philosophie. Elle prend en charge des éléments de vécu et dispose de concepts, comme le caractère obsessionnel ou l'hystérie, etc. À quoi on pourrait ajouter tout un ensemble de problématiques, comme les «manifestés » (ou symptômes) relevant du transgénérationnel. Il y a là des événements de vie qui ont un socle universel. C'est aussi de la littérature, tous les discours sur la folie! Le fou est encore plus proche de nous que l'homme sain (s'il existe) et on peut se reconnaître à certains égards dans son vécu (comme dans le théâtre de Shakespeare). Tout cela mérite d'être réinvesti dans le travail intellectuel de la recherche.

Avec ces remarques sur la folie, on semble bien loin de la philosophie. Encore que cette dernière est une discipline qui ne s'autorise que de la raison: et si le philosophe exerce le ministère de la raison, il lui faut bien définir cette dernière par opposition à son contraire qu'est censée être la folie. Plus fondamentalement, la philosophie a donc été d'emblée à l'arrière-plan de ma réflexion traductologique, mais tacitement et en retrait. Longtemps, j'ai tenu à dissocier ces deux modes de pensée dans mes travaux. Mais ces dernières années, j’ai consacré expressément plusieurs études aux rapports entre traduction et philosophie. Ainsi est-ce notamment la thématique dont traitait la conférence d'ouverture que j'ai eu l'honneur de prononcer au congrès annuel de l'Association canadienne de traductologie (ACT), à Ottawa le 23 mai 2009...

\section{- Vous associez la traductologie, la philosophie et la psychanalyse, pourquoi ?}

À mon sens, il y a trois disciplines réflexives: la philosophie, la psychanalyse et la traductologie (et sans doute pourrait-on en rajouter d'autres). L'idée est que ce sont des disciplines qui font retour sur ce qu'on vit, sur ce qu'on pense et ce qu'on fait. On en esquisse l'analyse réflexive rétrospectivement. Le philosophe passe son temps à faire de la méta-philosophie, c'est-à-dire qu'il se demande comment il philosophe. En psychanalyse, le «contre-transfert» est un travail réflexif que fait l'analyste sur lui-même et sur son rapport à l'autre (le patient). En traductologie, c'est plus élémentaire, mais tout de même comparable. Pour moi, l'objet d'analyse, c'est ce qui se passe dans la tête des traducteurs en général, et surtout du traducteur que je suis, ou des traducteurs que je forme. On est dans la réflexivité. Si j’ai longtemps insisté sur le triangle interdisciplinaire de la traductologie (linguistique, philosophie et psychologie), je dois dire que ce triangle est plutôt un carré, dans la mesure où il ne faut pas oublier la dimension littéraire et tout l'apport de la littérature comparée. Je reformulerais les choses ainsi: la traductologie se situe à l'articulation de la linguistique et de la littérature comparée, surplombée par la philosophie avec un soubassement en psychologie. À vrai dire, à y regarder de plus près, c'est de proche en proche toutes les sciences humaines et toutes les études culturelles qui sont finalement concernées, en sorte que la traductologie tend à prendre l'ampleur d'une anthropologie interdisciplinaire de la traduction. 


\section{BIBLIOGRAPHIE SÉLECTIVE}

\section{Principaux ouvrages}

Ladmiral, Jean-René et Lipiansky, Edmond Marc (1989): La Communication interculturelle. Paris: Armand Colin.

LAdmiral, Jean-René (1994): Traduire: théorèmes pour la traduction. Collection TEL, nº 246. Paris: Gallimard.

Ladmiral, Jean-René et Chateau, Dominique (1996): Critique et théorie. Paris: L'Harmattan. Ladmiral, Jean-René (2009) : Della traduzione: dall'estetica all'epistemologia, a cura di Antonio Lavieri. Collection Strumenti. Modène: Mucchi.

LAdmiral, Jean-René (2010): Jean-René Ladmiral, le dernier des archéotraductosaures, interviewé par l'ETIB. Collection Sources-Cibles, $\mathrm{n}^{\circ} 26$. Beyrouth: Université St-Joseph.

Ladmiral, Jean-René (2011): Aphorismes, édités et présentés par Mehdi Farrokhi. Collection Sources-Cibles, nº 22. Beyrouth/Bruxelles: Université St-Joseph/Éditions du Hazard.

\section{Choix d'articles et de contributions à des ouvrages collectifs}

Ladmiral, Jean-René (1980): Philosophie de la traduction et linguistique d'intervention. Lectures. 4-5:11-41.

LADMIRAL, Jean-René (1981): Éléments de traduction philosophique. Langue française. 51:19-34.

LAdMiral, Jean-René (1981): Entre les lignes, entre les langues. Revue d'esthétique. 1:67-77.

LAdMiral, Jean-René (1982): Traduction et psychosociologie. Meta. 27(2):196-206.

LADMiral, Jean-René (1984): Les 'théorèmes de la traduction'. In: Hildegunde BüHLER, dir. Der Übersetzer und seine Stellung in der Öffentlichkeit. Actes du X Congrès de la FIT. Vienne: Wilhelm Braumüller, 299-304.

Ladmiral, Jean-René (1986): Sourciers et ciblistes. Revue d'esthétique. 12:33-42.

Ladmiral, Jean-René (1987): Traductologiques. Le Français dans le monde. Numéro spécial: Retour à la traduction, dirigé par Marie-José Capelle, Francis Debyser et Jean-Luc GoEster, août-septembre 1987:18-25.

LAdMiral, Jean-René (1987): Pour la traduction dans l'enseignement des langues: «version» modernes des Humanités. Les Langues modernes. 1:9-21.

Ladmiral, Jean-René (1989): Traduire Freud: la langue, le style, la pensée. Actes des Cinquièmes Assises de la Traduction littéraire, 1988, Arles. Arles: Actes Sud-ATLAS, 152-155 et 168-171.

Ladmiral, Jean-René (1989): Pour une philosophie de la traduction. Revue de métaphysique et de morale. 1:5-22.

LAdMiral, Jean-René (1990): Pour une théologie de la traduction. TTR. 3(2):121-138.

LADMIRAL, Jean-René (1992): La traduction: philosophie d'une pratique. In: Christine Pagnoulle, dir. Les gens du passage. Liège: Université de Liège, 120-136.

Ladmiral, Jean-René (1995): Traduire, c'est-à-dire... Phénoménologies d’un concept pluriel. Meta. 40(3):409-420.

Ladmiral, Jean-René (1995): À partir de Georges Mounin: esquisse archéologique. TTR. 8(1):35-64.

LAdMiRal, Jean-René (1995): Que le traducteur est un réécrivain. Nouveaux Cahiers d'Allemand. 2(13):167-182.

Ladmiral, Jean-René (1996): Pour une psychologie de la traduction. In: Angelika Lauer, Heidrun Gerzymisch-Arbogast, Johann Haller, et al., dir. Übersetzungswissenschaft im Umbruch. Festschrift für Wolfram Wilss zum 70. Geburtstag. Tübingen: Gunter Narr Verlag, 27-35.

LADMIRAL, Jean-René (1997): Les quatre âges de la traductologie - Réflexions sur une diachronie de la théorie de la traduction. In: L'histoire et les théories de la traduction. Berne/Genève: ASTTI/ETI, 11-42.

Ladmiral, Jean-René (1998): Le prisme interculturel de la traduction. Palimpsestes. 11:15-30. 
Ladmiral, Jean-René (1998): Principes philosophiques de la traduction. In: André JaCoB, dir. Encyclopédie philosophique universelle. Volume 4: Le discours philosophique. Paris: Presses Universitaires de France, 977-998.

LADMIRAL, Jean-René (2002): De la linguistique à la littérature: la traduction. In: Jacques ANIs, André Eskenazi et Jean-François JeAndillou, dir. Le Signe et la lettre - Hommage à Michel Arrivé. Collection Sémantiques. Paris: L'Harmattan, 337-347.

LADMIRAL, Jean-René (2003): La traductologie au XXI siècle: de la linguistique à la psychologie. In: Traduire au XXIème siècle: Tendances et perspectives. Thessalonique: Université Aristote de Thessalonique, 336-346.

Ladmiral, Jean-René (2005): Le 'salto mortale’ de la déverbalisation. Meta. 50(2):473-487.

Ladmiral, Jean-René (2005): Formation des traducteurs et traduction philosophique. Meta. 50(1):96-106.

LADMiral, Jean-René (2006) : L'empire des sens. In: Mariane LedERER, dir. Le sens en traduction. Collection Cahiers Champollion. Paris/Caen: Lettres modernes, 109-125.

Ladmiral, Jean-René (2008): Pour un tournant œcuménique en théorie de la traduction. In: Christian BAlliu, dir. Traduire: un métier d'avenir. Vol. 1. Bruxelles: Les Éditions du Hazard, 11-32.

LAdmiral, Jean-René (2009): Traduction et philosophie. SEPTET - Des mots aux actes $N^{\circ} 2$ Traduction et philosophie du langage. 2:47-70.

Ladmiral, Jean-René (2009): La traduction: de la linguistique à la philosophie. In: Tatiana Milliaressi, dir. La traduction: philosophie, linguistique et didactique. Lille: Éditions du Conseil Scientifique de l'Université Charles-de-Gaulle, 3:29-32.

LAdMiral, Jean-René (2010): Sur le discours méta-traductif de la traductologie. Meta. 55(1):4-14.

LADMiral, Jean-René (2010): La traduction, phénomène interculturel et psycho-relationnel. Meta. 55(4):626-641.

Ladmiral, Jean-René (2010): Babel \& Logos. In: Hannelore Lee-Jahnke et Eric Prunc, Am Schnittpunkt von Philologie une Translationswissenschaft, Festschrift zu Ehren von Martin Forstner. Berne: Peter Lang, 171-201.

Ladmiral, Jean-René (2011): Approche méta-théorique. In: Christian Berner et Tatiana Milliaressi. La traduction: philosophie et tradition. Collection Philosophie \& linguistique. Villeneuve d'Ascq: Presses Universitaires du Septentrion, 23-40.

\section{Hommages}

Traduire «Traduire». Autour des travaux de Jean-René Ladmiral (2004). Colloque organisé par l'Institut supérieur d'interprétation et de traduction, Institut Catholique de Paris, 27 mai 2004. Transversalités. 92.

D’AméLio, Nadia et Hewson, Lance, dir. (2011): J'ai dit la traductologie sans que j'en susse rien. Hommages à Jean-René Ladmiral. Université de Mons, Éditions du CIPA.

LAutel-Ribstein, Florence et Fort, Camille, dir. (2012): Jean-René Ladmiral: une ceuvre en mouvement. Actes du colloque international organisé les 3 et 4 juin 2010 à l'Université Paris-Sorbonne (SEPTET). Des mots aux actes en traduction $n^{\circ}$ 3. Perros-Guirec: Éditions Anagrammes. 